\title{
Some Special Features of Finite-Source Retrial Queues with Collisions, an Unreliable Server and Impatient Customers in the Orbit
}

\author{
János Sztrik ${ }^{(\bowtie)}$ (1) and Ádám Tóth®(]) \\ Faculty of Informatics, University of Debrecen, Debrecen, Hungary \\ \{sztrik.janos, toth.adam\}@inf .unideb.hu
}

\begin{abstract}
The goal of the paper is to study a finite-source retrial queuing system with collisions and customers' impatience behavior in the orbit. The situation when an incoming customer from the orbit or from the source finds the server busy causes a collision and both requests are directed toward the orbit. It is assumed that every request in the source is eligible to generate customers whenever the server is failed but these requests immediately go into orbit. A customer after some waiting in the orbit can depart without fulfilling its service requirement these are the socalled impatient/reneging/abandoned customers. In that case it returns to the source. A customer who is under service when the server fails is also sent to the orbit. The source, service, retrial, impatience, operation and repair times are supposed to be independent of each other. The novelty of the investigation is to carry out a sensitivity analysis comparing various distributions of impatience time of customers on the performance measures such as mean number of customers in the orbit, mean waiting time of an arbitrary, successfully served and reneging customers, probability of abandonment, server utilization, etc.
\end{abstract}

Keywords: Finite-source queuing system - Retrial queues ·

Collisions - Server breakdowns and repairs - Impatient customers ·

Stochastic simulation

\section{Introduction}

Impatience of the customers is a natural phenomenon and an interesting topic in queueing theory. The process of reneging and balking is extensively studied by many researchers, for example in $[1-10,13,14,20,24]$. Whenever an arriving customer decides not to enter the system, which is called balking while in reneging a customer in the system after waiting for some time leaves the system

The research of both authors was supported by the construction EFOP-3.6.3-VEKOP16-2017-00002. The project was supported by the European Union, co-financed by the European Social Fund.

(C) Springer Nature Switzerland AG 2021

A. Dudin et al. (Eds.): ITMM 2020, CCIS 1391, pp. 1-15, 2021.

https://doi.org/10.1007/978-3-030-72247-0_1 
without being served. In our investigated model reneging customers are considered. Queuing systems with repeated calls may competently describe major telecommunication systems, such as telephone switching systems, call centers, CSMA-based wireless mesh networks in frame level. The main feature of retrial queueing system is that customers remain in the system even if it is unable to find idle service unit and after some random time it attempts to reach the service facility again. Speaking of communication systems where the available channels or other facilities are very limited thus users (sources) usually need to fight for these resources. This results a high possibility of conflict because several sources may launch uncoordinated attempts producing collisions. In these cases the loss of transmission takes place and it is necessary to ensure of the process of retransmission. So evolving efficient procedures for preventing conflict and corresponding message delay is essential. In case of a collision both calls, the one under service and the newly arriving one go to the orbit. A review of results on finite-source retrial queues with collision and unreliable server has been published in [18]. In many papers of retrial queueing literature the service unit is assumed to be available steadily. But these assumptions are quite unrealistic because in real life applications of these systems can break down, different types of problems can arise like power outage, human error or other failures. Various factors have effect on the transmission rate of the wireless channel in a wireless communication scenario and these are apt to suffer transmission failure, interruptions throughout transferring the packets. Investigating retrial queueing systems with random server breakdowns and repairs has a great importance as the operation of non-reliable systems modifies system characteristics and performance measures. In this paper, we assume that in the case of a failure of the server, the request generation from the source continues and calls go to the orbit. Moreover, a customer who is under service when the server fails is also sent to the orbit.

The novelty of this investigation is to carry out sensitivity analysis using different distributions of impatient calls on performance measures. Different Figures help to understand the special features of the system. The model is a generalization of [21] and a continuation of the works [12,22].

The aim of the present paper is to show some special features of finite-source retrial queuing systems with impatient customers in the orbit. In general we could see that the steady-state distribution of the number of customers in the service facility can be approximated by a normal distribution with given mean and variance. By the help of stochastic simulation several systems are analyzed showing directions for further analytic investigations. Tables and Figures are collected to illustrate unexpected properties of these systems.

\section{System Model}

A retrial queueing system of type $M / M / 1 / / N$ is considered with a non-reliable server and impatient customers. In the finite-source $N$ customers reside and each of them is able to generate calls towards the server with rate $\lambda / N$ so the interrequest time is exponential with parameter $\lambda / N$. A customer cannot generate a 
new call until the previous call returns to the source. Every incoming customer has a random impatience time which determines how much time the customer spends in the orbit without getting its service requirement. Exceeding this time results that the customer no longer waits for the service unit and departs without being served properly. This random time follows gamma, hypo-exponential, hyper-exponential, Pareto and lognormal distribution with different parameters but with the same mean value. If an arriving customer either from the source or from the orbit finds the server in idle state its service starts immediately. The service times of the customers is assumed to be exponentially distributed with parameter $\mu$. After its successful service customers return to the source. Encountering the service unit in a busy state the arriving customer causes a collision with the call under service and both enter the orbit. After an exponentially distributed time with parameter $\sigma / N$ customers located in the orbit make another attempt to get into the service. The server is not reliable so from time to time can break down. The lifetime is an exponentially distributed random variable with parameter $\gamma_{0}$ in case of an idle server and $\gamma_{1}$ when the server is busy. The repair process starts immediately upon the breakdown which also follows an exponential distribution with parameter $\gamma_{2}$. If server failure takes place during the service of a customer it is transferred to the orbit. Furthermore, in the case of a failure, we can distinguish two options. Namely, the failure either stops entering new customers from the source or allows them to go to the orbit. Usually, we treat the system with the latter option if the other one is not stated. The source, service, retrial, impatience, operation, and repair times are supposed to be independent of each other.

\section{Simulation Case Studies}

The simulation approach is a very important method that helps us in performance modeling when the system is too complicated to investigate with the help of other standard methods, like analytical, numerical, or asymptotic ones. For the interested readers we list some of the most important works, such as $[11,15-17,19,23]$.

In this section first, we deal with exponentially distributed impatience time which helps us to check our simulation results with the help of those we got using MOSEL (MOdeling, Specification, and Evaluation Language), published in [12]. As soon as we realized that the simulation program operates correctly we can investigate the effect of the distribution of impatience time on the performance measures as we will do in the second part. One of the advantages of the simulation that we can make a difference between abandoned and successfully served requests as we show in our examples. Reading the papers dealing with abandonment we noticed that mainly the distribution of the customers in the system has been investigated and then using the Little-formula the mean response time of an arbitrary customer has been obtained. There are no performance measures for different types of customers. 


\subsection{Exponentially Distributed Impatience Times}

In these cases we would like to show the effect of the impatience rate on the distribution of the number of customers in the system. All the random variables mentioned above are exponentially distributed (Table 1).

Table 1. Different impatience rates

\begin{tabular}{l|l|l|l|l|l|l|l|l}
\hline & $\mathrm{N}$ & $\lambda / N$ & $\sigma / N$ & $\gamma_{0}$ & $\gamma_{1}$ & $\gamma_{2}$ & $\mu$ & $\tau$ \\
\hline Case 1 & 100 & 0.01 & 0.1 & 0.1 & 0.1 & 1 & 1 & $1 \mathrm{E}-10$ \\
\hline Case 2 & 100 & 0.01 & 0.1 & 0.1 & 0.1 & 1 & 1 & 0.000001 \\
\hline Case 3 & 100 & 0.01 & 0.1 & 0.1 & 0.1 & 1 & 1 & 0.0001 \\
\hline Case 4 & 100 & 0.01 & 0.1 & 0.1 & 0.1 & 1 & 1 & 0.001 \\
\hline Case 5 & 100 & 0.01 & 0.1 & 0.1 & 0.1 & 1 & 1 & 0.01 \\
\hline Case 6 & 100 & 0.01 & 0.1 & 0.1 & 0.1 & 1 & 1 & 0.1 \\
\hline Case 7 & 100 & 0.01 & 0.1 & 0.1 & 0.1 & 1 & 1 & 1 \\
\hline Case 8 & 100 & 0.01 & 0.1 & 0.1 & 0.1 & 1 & 1 & 5
\end{tabular}

The results are understandable and illustrate what we expected, namely the higher the impatience rate the less the number of customers in the system see Fig. 1. However, we are able to give other measures which show some unexpected features of the system. It should be mentioned that we need some special parameter set up so that these cases should happen. We have some experience to find this setup from our previous works.

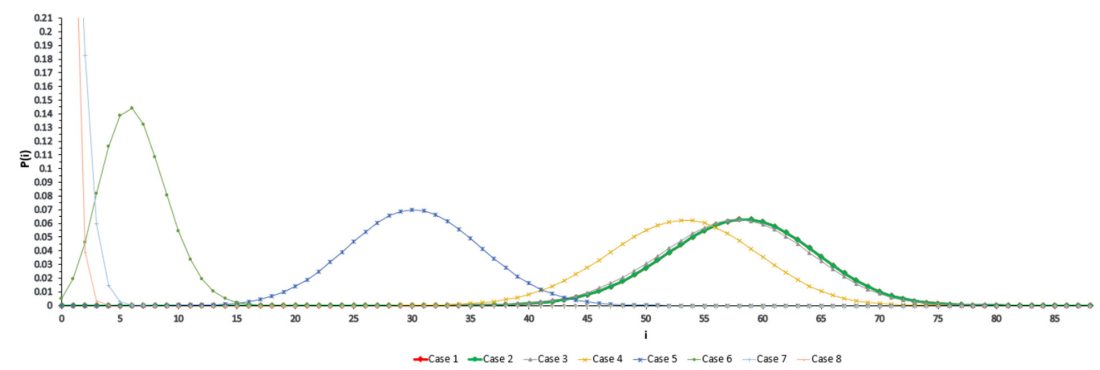

Fig. 1. Distribution of number of customers for different impatience rates

In the following we give detailed estimations for the different type of customers, namely successfully served and abandoned requests. In our opinion only the simulation can help us to receive these measures. In Tables 2, 3, 4 we can see some special features of these systems for which the notations are given in Table 5 . 
Table 2. Estimations 1

\begin{tabular}{l|l|l|l|l|l|l|l|l|l}
\hline & $\mathrm{E}(\mathrm{NS})$ & $\mathrm{E}(\mathrm{O})$ & $\mathrm{E}(\mathrm{T})$ & $\mathrm{E}(\mathrm{TS})$ & $\mathrm{E}(\mathrm{TA})$ & $\mathrm{E}(\mathrm{W})$ & $\mathrm{E}(\mathrm{WS})$ & $\mathrm{E}(\mathrm{WA})$ & $\mathrm{E}(\mathrm{WAO})$ \\
\hline Case 1 & 57.9829 & 57.5627 & 137.9729 & 137.9729 & 0.0000 & 136.9730 & 136.9730 & 0.0000 & 0.0000 \\
\hline Case 2 & 57.9771 & 57.5570 & 137.9197 & 137.9182 & 148.6876 & 136.9203 & 136.9188 & 147.7836 & 20.1735 \\
\hline Case 3 & 57.4366 & 57.0166 & 134.9306 & 134.7932 & 145.0371 & 133.9441 & 133.8056 & 144.1288 & 20.3340 \\
\hline Case 4 & 52.9963 & 52.5786 & 112.7086 & 111.5587 & 121.8498 & 111.8203 & 110.6612 & 121.0345 & 19.9312 \\
\hline Case 5 & 30.2477 & 29.8485 & 43.3468 & 39.3924 & 48.6341 & 42.7747 & 38.7689 & 48.1307 & 16.3978 \\
\hline Case 6 & 6.3188 & 5.9797 & 6.7437 & 3.6688 & 8.4876 & 6.3818 & 3.1664 & 8.2053 & 6.3764 \\
\hline Case 7 & 0.9897 & 0.6868 & 0.9996 & 0.5514 & 1.1972 & 0.6937 & 0.0633 & 0.9716 & 0.9432 \\
\hline Case 8 & 0.4365 & 0.1398 & 0.4384 & 0.4849 & 0.4187 & 0.1404 & 0.0030 & 0.1987 & 0.1974 \\
\hline
\end{tabular}

Table 3. Estimations 2

\begin{tabular}{l|l|l|l|l|l|l|l|l|l}
\hline $\mathrm{E}(\mathrm{ST})$ & $\mathrm{E}(\mathrm{STS})$ & $\mathrm{E}(\mathrm{STA})$ & $\mathrm{E}(\mathrm{STSI})$ & $\mathrm{E}(\mathrm{STSUI})$ & $\mathrm{Pa}$ & Pao & Us & UsS & UsA \\
\hline 0.9999 & 0.9999 & 0.0000 & 0.8606 & 0.1394 & 0.0000 & 0.0000 & 0.4202 & 0.4200 & 0.0000 \\
\hline 0.9994 & 0.9994 & 0.9040 & 0.8603 & 0.1394 & 0.0001 & 0.0739 & 0.4201 & 0.4200 & 0.0001 \\
\hline 0.9866 & 0.9876 & 0.9083 & 0.8474 & 0.1403 & 0.0134 & 0.0777 & 0.4200 & 0.4149 & 0.0052 \\
\hline 0.8883 & 0.8975 & 0.8152 & 0.7489 & 0.1487 & 0.1117 & 0.0906 & 0.4177 & 0.3749 & 0.0428 \\
\hline 0.5721 & 0.6235 & 0.5034 & 0.4095 & 0.2140 & 0.4279 & 0.1874 & 0.3992 & 0.2489 & 0.1504 \\
\hline 0.3619 & 0.5024 & 0.2823 & 0.1118 & 0.3907 & 0.6381 & 0.4272 & 0.3391 & 0.1703 & 0.1688 \\
\hline 0.3059 & 0.4881 & 0.2256 & 0.0162 & 0.4718 & 0.6940 & 0.5318 & 0.3029 & 0.1479 & 0.1553 \\
\hline 0.2980 & 0.4819 & 0.2200 & 0.0039 & 0.4780 & 0.7020 & 0.5426 & 0.2968 & 0.1431 & 0.1538 \\
\hline
\end{tabular}

It is easy to check that the mean response/waiting and total service time of an arbitrary customer can be obtained by the help of law of total expectation. Furthermore, the Little-formulas are also valid.

Let us make some comments concerning the results. If we take a closer look at the mean waiting time of an abandoned customer $E(W A)$ and the conditional mean waiting time $E(W A O)$ of those abandoned customers who never left the orbit we can see one of the unexpected features. Namely, one might think that they should be around the mean of the assumed impatience time, in this case $1 / \tau$. But as we can observe the estimations are much less in the rows $2-5$ of Table 2. Our explanation is the following: since the impatience rates are small the customers abandon very rarely and in the realizations only the short durations happen. Thus the sample mean of these few durations cannot be considered as the true estimation of the hypothetical expectation $E(W A O)$.

In Table 3 the mean total service time of a successful customer $E(S T S)$ decreases but the mean of the uninterrupted service time of a successful request increases. We have similar explanation, namely as more and more request abandons the system less and less customer needs service. Thus the number of collisions decreases and as a consequence the uninterrupted service times increase but their mean is less than the expectation of the hypothetical service time since only the shorter durations are considered. The behavior of the probabilities are reasonable, but again the utilization of the server with respect to the abandoned 
Table 4. Estimations 3

\begin{tabular}{l|l|l|l|l|l|l|l}
\hline $\operatorname{Var}(\mathrm{NS})$ & $\operatorname{Var}(\mathrm{T})$ & $\operatorname{Var}(\mathrm{TS})$ & $\operatorname{Var}(\mathrm{TA})$ & $\operatorname{Var}(\mathrm{W})$ & $\operatorname{Var}(\mathrm{WS})$ & $\operatorname{Var}(\mathrm{WA})$ & $\operatorname{Var}(\mathrm{ST})$ \\
\hline 40.007 & 21911.908 & 21911.908 & 0.000 & 21657.862 & 21657.862 & 0.000 & 1.000 \\
\hline 40.124 & 21881.572 & 21881.345 & 23376.692 & 21627.907 & 21627.682 & 23112.600 & 0.999 \\
\hline 40.251 & 20963.887 & 20960.814 & 21088.319 & 20719.418 & 20716.321 & 20844.001 & 0.975 \\
\hline 40.608 & 14752.973 & 14728.385 & 14854.131 & 14571.818 & 14547.085 & 14672.623 & 0.805 \\
\hline 32.106 & 2326.767 & 2264.987 & 2360.505 & 2287.902 & 2225.639 & 2321.008 & 0.386 \\
\hline 7.570 & 65.502 & 44.016 & 69.292 & 63.994 & 41.980 & 67.298 & 0.233 \\
\hline 0.980 & 0.999 & 0.387 & 1.141 & 0.867 & 0.118 & 0.945 & 0.205 \\
\hline 0.344 & 0.215 & 0.235 & 0.204 & 0.036 & 0.001 & 0.039 & 0.199 \\
\hline
\end{tabular}

Table 5. Notation for the estimations

$\mathrm{E}(\mathrm{NS})$ : mean number of customers in the system

$\mathrm{E}(\mathrm{T})$ : mean sojourn time of an arbitrary customer

$\mathrm{E}(\mathrm{TS})$ : mean sojourn time of a successfully served customer

$\mathrm{E}(\mathrm{TA})$ : mean sojourn time of a reneging customer

$\mathrm{E}(\mathrm{O})$ : mean number of customers in the orbit

$\mathrm{E}(\mathrm{W})$ : mean waiting time of an arbitrary customer

$\mathrm{E}(\mathrm{WS})$ : mean waiting time of a successfully served customer

$\mathrm{E}(\mathrm{WA})$ : mean waiting time of a abandoned customer

$\mathrm{E}(\mathrm{ST})$ : mean total service time of an arbitrary customer

$\mathrm{E}(\mathrm{STS})$ : mean total service time of a successfully served customer

$\mathrm{E}(\mathrm{STA})$ : mean total service time of a reneging customer

Pa: probability of abandonment

Us: server utilization

$\operatorname{Var}(\mathrm{NS})$ : variance of number of customers in the system

$\operatorname{Var}(\mathrm{T})$ : variance of sojourn time of an arbitrary customer

$\operatorname{Var}(\mathrm{TS})$ : variance of sojourn time of a successfully served customer

$\operatorname{Var}(\mathrm{TA})$ : variance of sojourn time of a reneging customer

$\operatorname{Var}(\mathrm{W})$ : variance of waiting time of an arbitrary customer

$\operatorname{Var}(\mathrm{WS})$ : variance of waiting time of a successfully served customer

$\operatorname{Var}(\mathrm{WA})$ : variance of waiting time of a reneging customer

$\operatorname{Var}(\mathrm{ST})$ : variance of total service time of an arbitrary customer

$\operatorname{Var}(\mathrm{STS})$ : variance of total service time of a successful customer

$\operatorname{Var}(\mathrm{STA})$ : variance of total service time of an abandoned customer

Pao: conditional probability that an abandoned customer never

leaves the orbit

$\mathrm{E}(\mathrm{WAO})$ : mean waiting time of an abandoned customer who never leaves the orbit

E(STSI): mean total interrupted service time of a successful customer

E(STSUI): mean total uninterrupted service time of a successful customer

UsA: server utilization of an abandoned customer

UsS: server utilization of a successfully served customer 
customers $U s A$ is surprising. We cannot explain why at the beginning it increases then decreases.

\subsection{Generally Distributed Impatience Times}

Our aim is to examine how the different distributions of impatience of calls have an effect on the performance measure when the mean and variance are equal, respectively. The investigations are divided into two parts depending on the squared coefficient of variation.

\section{Squared Coefficient of Variation is Greater than One}

In the first part Table 6 shows the parameters of distinct distributions. The parameters are chosen in such a way that the squared coefficient of variation would be greater than one. For comparison hyper-exponential, gamma, lognormal and Pareto distributions are used besides the case when the impatience time is constant. Our simulation program is equipped with random number generators and these functions need input parameters which are different in every distribution.

Numerical values of model parameters are the following:

$$
N=100 \quad \lambda / N=0.01 \quad \gamma_{0}=0.1 \quad \gamma_{1}=0.1 \quad \gamma_{2}=1 \quad \sigma / N=0.1 \quad \mu=1
$$

Table 6. Parameters of impatience distributions, squared coefficient of variation is greater than one

\begin{tabular}{l|l|l|l|l}
\hline Distribution & Gamma & Hyper-exponential & Pareto & Lognormal \\
\hline Parameters & $\alpha=0.390625$ & $p=0.33098$ & $\alpha=2.1792$ & $m=5.57973$ \\
& $\beta=0.0007813$ & $\begin{array}{l}\lambda_{1}=0.00132 \\
\lambda_{2}=0.00268\end{array}$ & $k=270.56302$ & $\sigma=1.12684$ \\
\hline Mean & 500 & & \\
\hline Variance & 640000 & & \\
\hline Squared coefficient of variation & 2.56 & & \\
\hline
\end{tabular}

Figure 2 shows the comparison of steady-state distribution of the number of customers in the system. Taking a closer look on the results all the curves correspond to a normal distribution, the explanation can be found in paper [18]. However, this figure clearly displays the contrast among the applied distributions. Although the shape of the curves is almost the same the average number of customers in the system varies a little bit especially in case of Pareto distribution and when the impatience time of calls is constant the mean is greater compared to the others.

The mean response time of different types of customer is shown in function of arrival intensity on Figs. 3, 4, 5. Figure 4 illustrates how the mean response time of impatient customers changes. The mean waiting time in the orbit should 


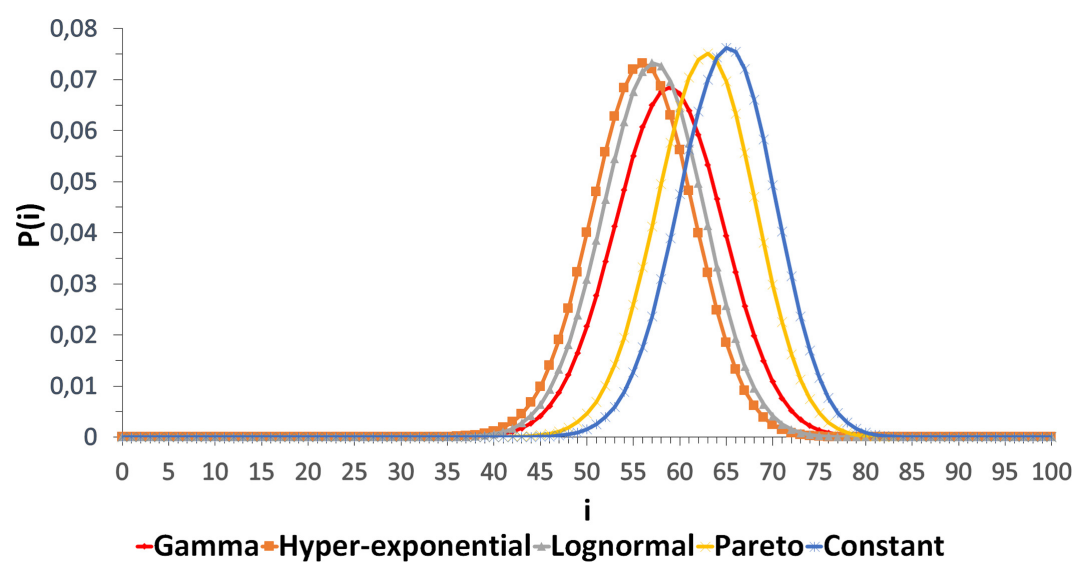

Fig. 2. Comparison of steady-state distributions

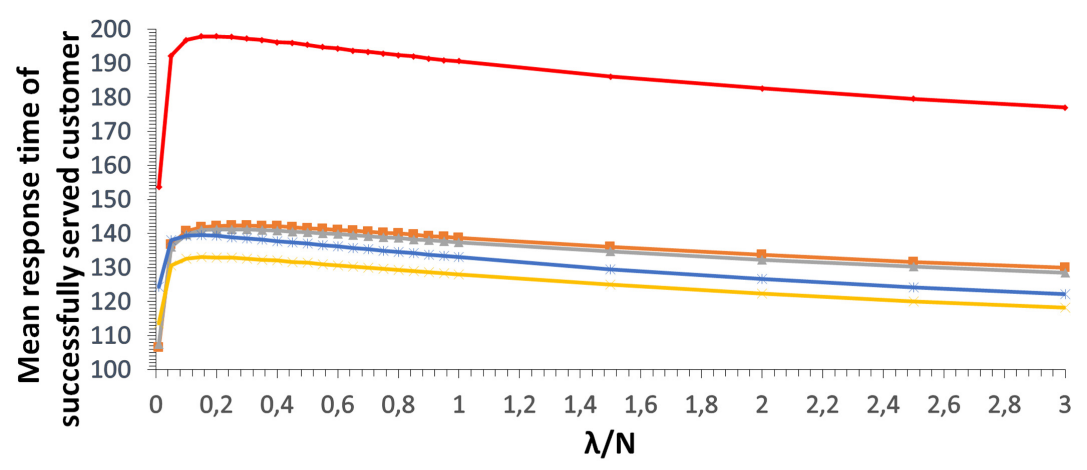

-Gamma =Hyper-exponential =Lognormal -Pareto -Constant

Fig. 3. Mean response time vs. arrival intensity using various distributions

be constant, due to the constant impatient time of a customer. Of course, Fig. 5 can be obtained with the help of the law of total expectation, too. Interestingly, differences can be observed even though the first two moments are equal, respectively. Results clearly illustrate the effect of various distributions. Highest values are experienced at gamma distribution in the case of successful customers, but in the case of impatient calls, constant impatience time gives the greatest values. Despite the increasing arrival intensity the maximum property characteristic of finite-source retrial queueing systems occurs under suitable parameter settings as we mentioned in [18].

Figure 6 demonstrates how the probability of abandonment of a customer changes with the increment of the arrival intensity. Under probability of abandonment we mean the probability of that a customer leaves the system without getting its full service requirement (through the orbit). After a slow increase of the value of this performance measure it stagnates which is true for every used 


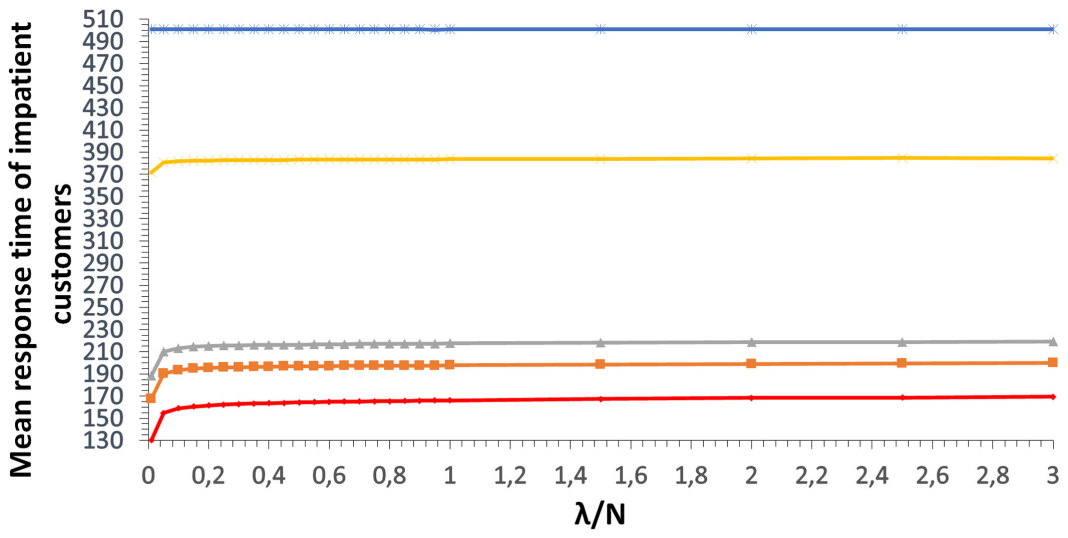

-Gamma -Hyper-exponential =-Lognormal - Pareto -Constant

Fig. 4. Mean response time vs. arrival intensity using various distributions

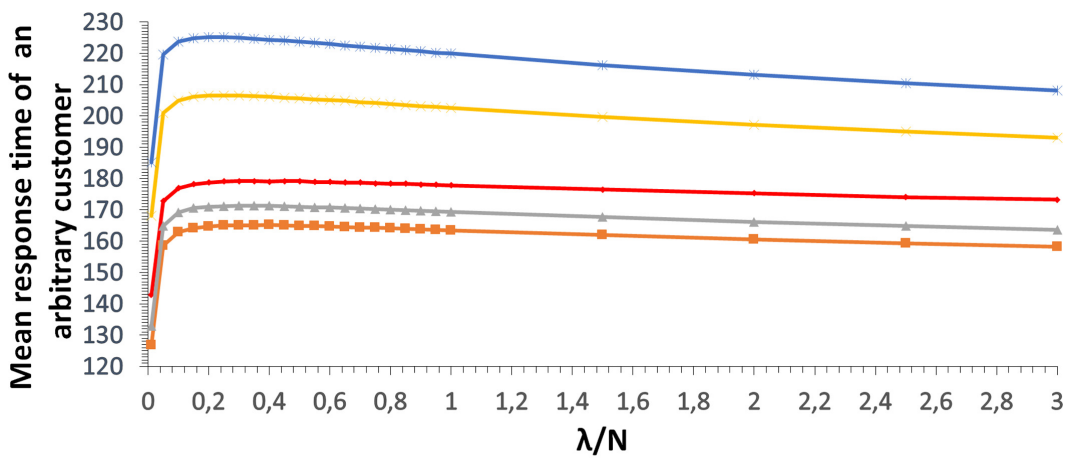

-Gamma $=$ Hyper-exponential =Lognormal - Pareto -Constant

Fig. 5. Mean response time vs. arrival intensity using various distributions

distributions of impatience of calls but they differ significantly from each other. At gamma distribution the tendency of leaving the system earlier is much higher than the others especially compared to at constant mean of impatience of calls. Here the disparity is much higher among the applied distributions compared to the previous Figures. An explanation of this feature could be the following: if the squared coefficient of variation is greater than one the gamma distribution takes small values with great probability, so the customers leave the system quite early and thus the probability of abandonment is high.

Figure 7 is related to the total utilization of server versus arrival intensity. Total utilization contains every service time including the interrupted ones no matter whether a call departed from the service unit or from the orbit. By examining closely the Figure we find prominent results when gamma distribution is applied and regarding the others the received values are almost identical. With 


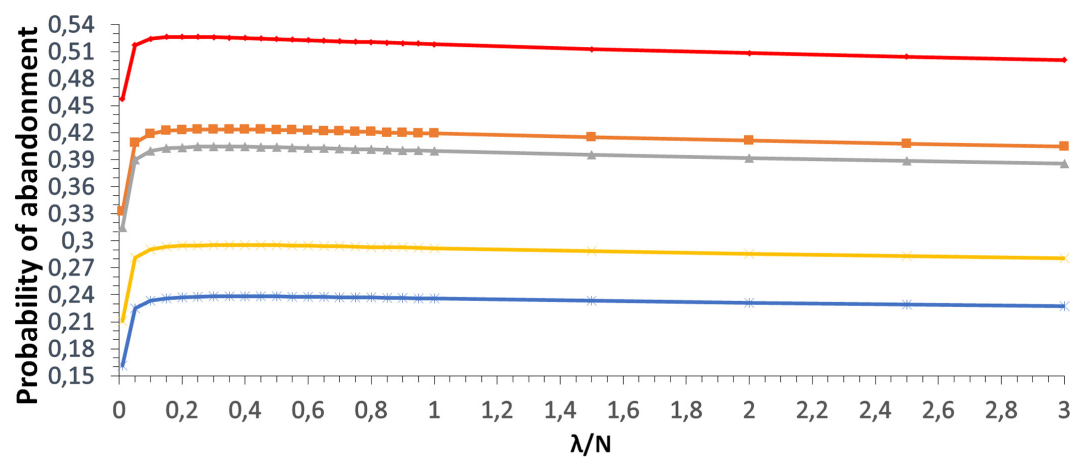

-Gamma -Hyper-exponential - Lognormal-Pareto-Constant

Fig. 6. Probability of abandonment of a customer vs. arrival intensity using various distributions

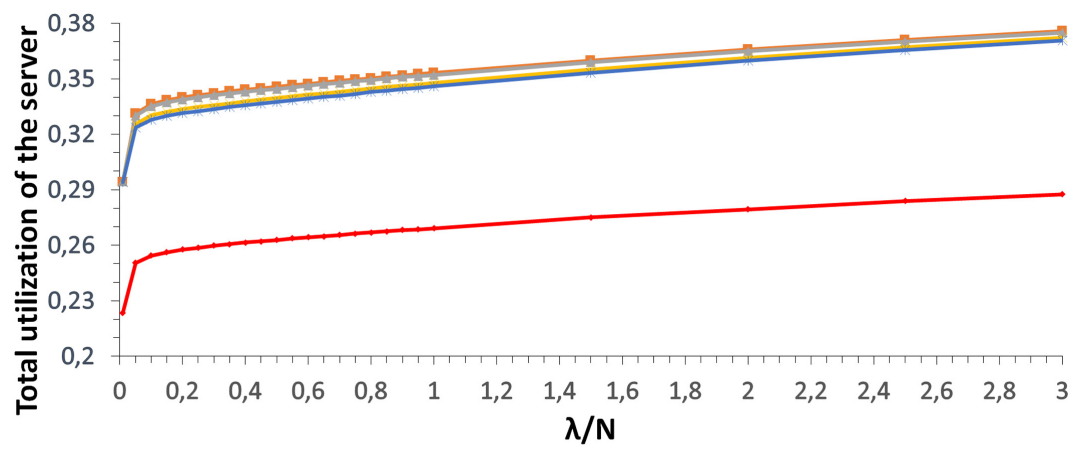

-Gamma-Hyper-exponential-Lognormal-Pareto-Constant

Fig. 7. Total utilization of server vs. arrival intensity using various distributions

the increment of arrival intensity the total utilization of the service unit increases as well. Here the explanation is the same as in the previous Figure, that is there are less customers in the system and hence the utilization is the smallest.

\section{Squared Coefficient of Variation is Less than One}

We carried the simulation in the case when the squared coefficient of variation is less than one, the mean is the same as before. The differences are noticeable but not as big as in the previous cases.

After viewing the above outcomes and figures we are intrigued to know how the operation of the system changes if another parameter setting is used. To do so we modify the parameters in order the squared coefficient of variation to be less than one so hyper-exponential is exchanged for hypo-exponential distribution. Table 7 contains the modified parameter setting of distribution of impatience of calls. Other parameters remain unchanged (see Table 6). 
Table 7. Parameters of impatience distributions, squared coefficient of variation is less than one

\begin{tabular}{l|l|l|l|l}
\hline Distribution & Gamma & Hypo-exponential & Pareto & Lognormal \\
\hline Parameters & $\alpha=1.47059$ & $\mu_{1}=0.01$ & $\alpha=2.5718$ & $m=5.9552$ \\
& $\beta=0.002941$ & $\mu_{2}=0.0025$ & $k=305.5844$ & $\sigma=0.72027$ \\
\hline Mean & 500 & & \\
\hline Variance & 170000 & & \\
\hline Squared coefficient of variation & 0.68 &
\end{tabular}

First, Fig. 8 represents the steady state distribution of the number of customers in the system. Analyzing the curves in more detail they are much closer to each other as on Fig. 2. Differences appear among the applied distributions with this parameter setting, too. As regard to the values with these parameters the mean number of customer is higher in case of every distribution. We think that this feature is due to the smaller variance of impatience time and customers stay in the orbit for a longer time since in the realization of the simulation there are less early abandonment.

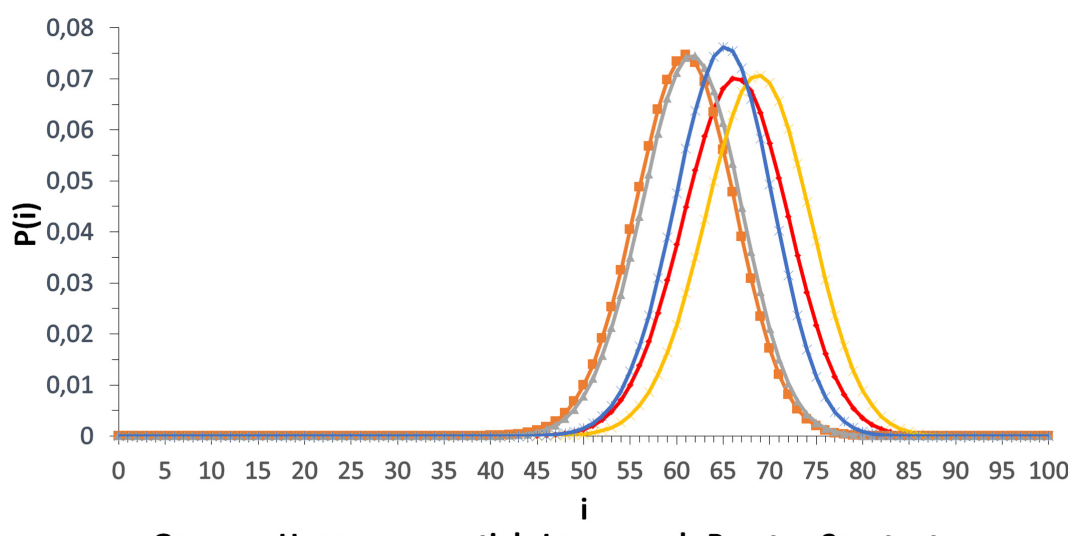

-Gamma =-Hypo-exponential - Lognormal-Pareto-Constant

Fig. 8. Comparison of steady-state distributions

The next Figures show the mean response time of different types of customers in function of arrival intensity. Examining Figures the same tendency can be seen as on the previous corresponding Figures but differences can still be discovered especially in case of gamma distribution. These Figures also reveal that successful customers on the average spend less time in the system compared to the previous parameter setting but the impatient and arbitrary ones spend longer time in the system (Figs. 9, 10 and 11). 


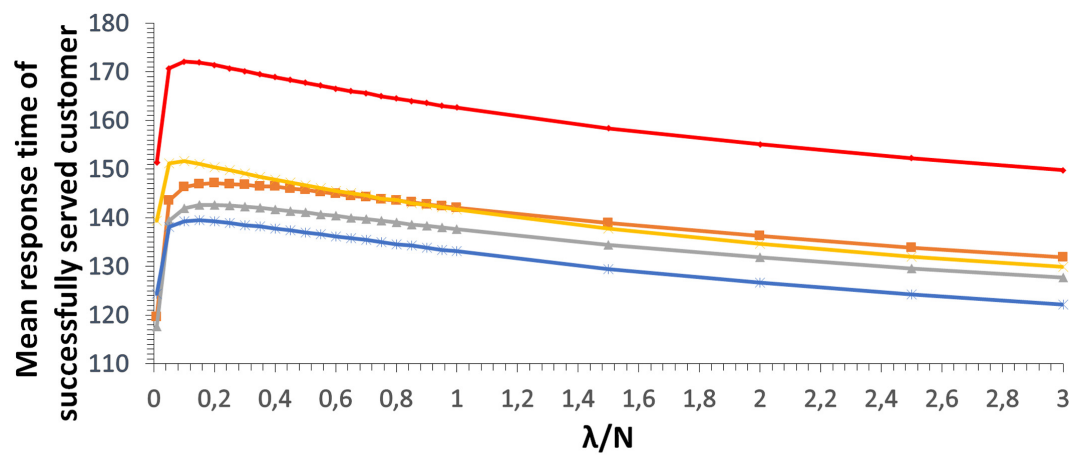

-Gamma $=$ Hypo-exponential $=$ Lognormal - Pareto -Constant

Fig. 9. Mean response time vs. arrival intensity using various distributions

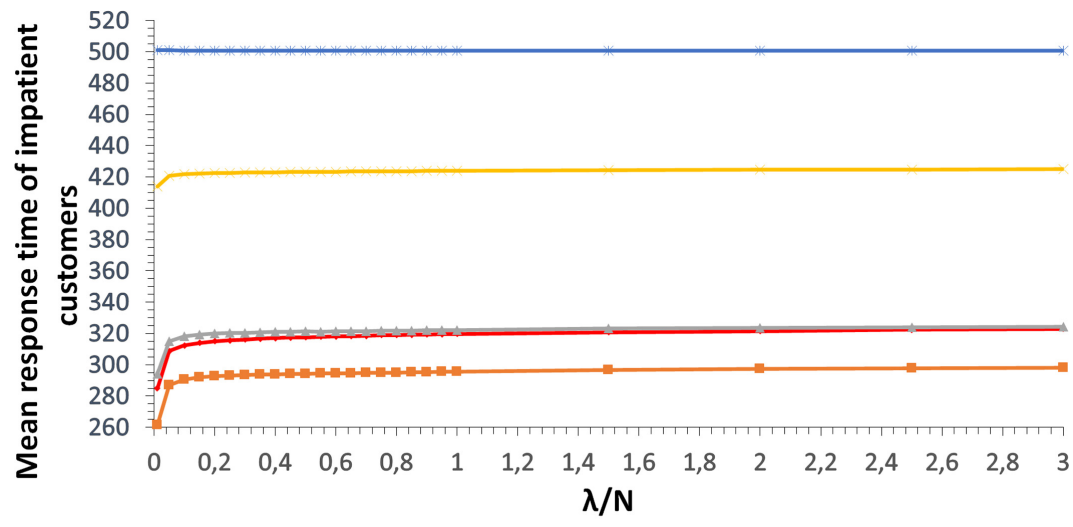

-Gamma =Hypo-exponential =Lognormal -Pareto -Constant

Fig. 10. Mean response time vs. arrival intensity using various distributions

Figure 12 demonstrates the probability of abandonment of a customer versus arrival intensity. Not surprisingly after seeing the previous two Figures the difference of achieved values are relatively far from each other, disparity is still present among the applied distributions. We can observe that the probability of abandonment is less than in the case of previous case, see Fig. 6. Our explanation is the same as in the case of number of customers in the system.

Lastly, on Fig. 13 the running parameter (value of x-axis) is the arrival intensity and value of $y$-axis is the total utilization of the server. Among the lines there are not so significant differences, they coincide with each other meaning that the utilization is almost the same except in case of Pareto and gamma distribution where the utilization of service unit is significantly less. 


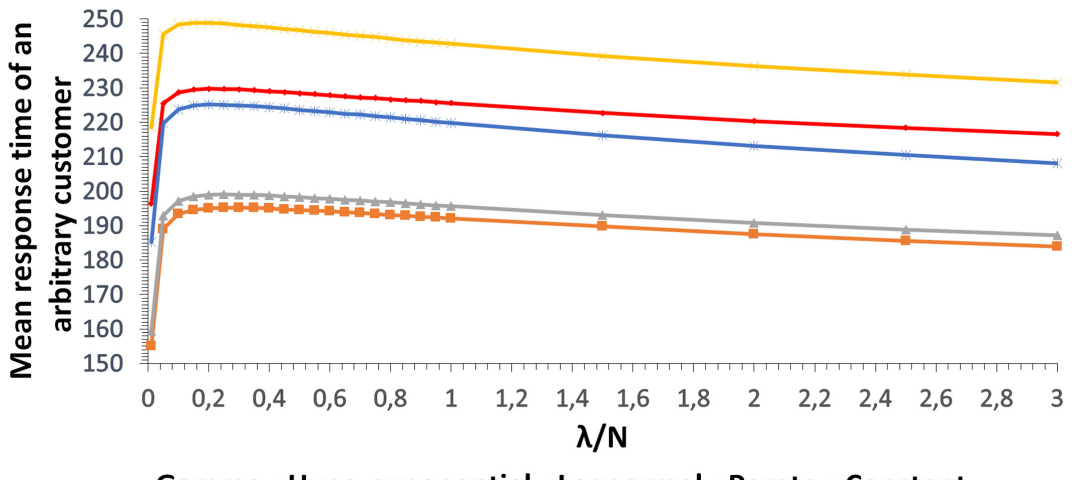

-Gamma =Hypo-exponential =Lognormal -Pareto -Constant

Fig. 11. Mean response time vs. arrival intensity using various distributions

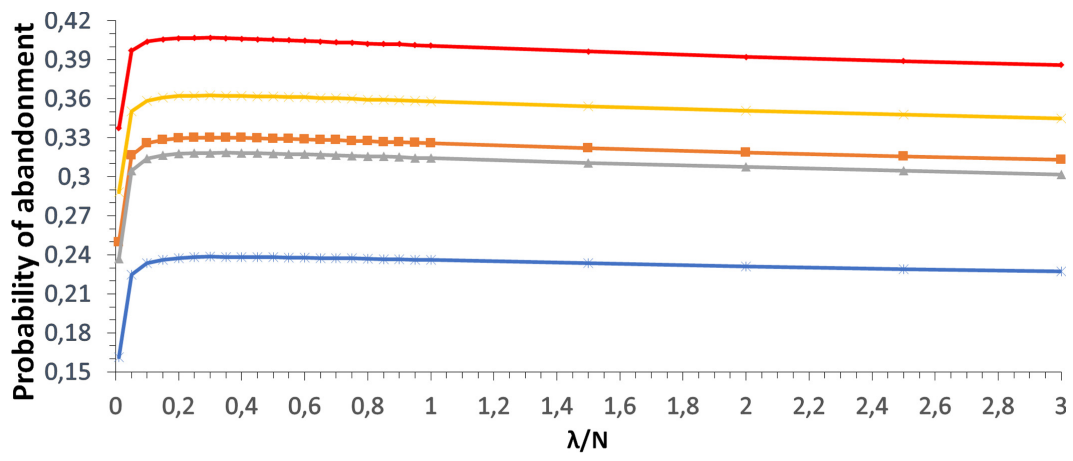

\section{-Gamma -Hypo-exponential =-Lognormal - Pareto -Constant}

Fig. 12. Probability of abandonment of a customer vs. arrival intensity using various distributions

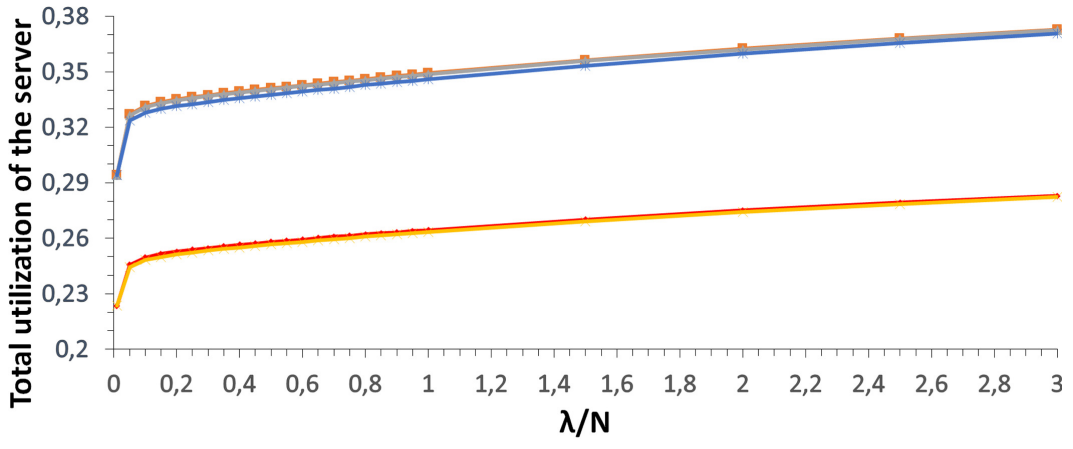

-Gamma =Hypo-exponential =Lognormal -Pareto-Constant

Fig. 13. Total utilization of server vs. arrival intensity using various distributions 


\section{Conclusion}

In this paper a finite-source retrial queueing system is presented with a nonreliable server, collisions and impatient customers. The obtained results fully demonstrated how essential is the distribution of impatience of calls because it has a great influence on the system characteristics despite the fact that the mean and the variance are the same, respectively. Figures in connection several performance measures, for example the probability of abandonment clearly assure this phenomenon. Results evidently indicated the distinction is noticeable and significant among the performance measures when the squared coefficient of variation is greater than one and moderate when it is less than one. In the future we would like to deal with more distributions to expand our investigation and examine the performance measures when the distribution of service time is not exponential. We also would like to analyze systems of two-way communications with impatient customers.

\section{References}

1. Adan, I., Hathaway, B., Kulkarni, V.: On first-come, first-served queues with two classes of impatient customers. Queueing Syst. 91(1-2), 113-142 (2019)

2. Aissani, A., Lounis, F., Hamadouche, D., Taleb, S.: Analysis of customers' impatience in a repairable retrial queue under postponed preventive actions. Am. J. Math. Manag. Sci. 38(2), 125-150 (2019)

3. Artalejo, J.R., Pla, V.: On the impact of customer balking, impatience and retrials in telecommunication systems. Comput. Math. Appl. 57(2), 217-229 (2009)

4. Danilyuk, E., Moiseeva, S., Nazarov, A.: Asymptotic analysis of retrial queueing system $M / G I / 1$ with collisions and impatient calls. In: Dudin, A., Nazarov, A., Moiseev, A. (eds.) ITMM 2019. CCIS, vol. 1109, pp. 230-242. Springer, Cham (2019). https://doi.org/10.1007/978-3-030-33388-1_19

5. Danilyuk, E., Moiseeva, S., Sztrik, J.: Asymptotic analysis of retrial queueing system $M / M / 1$ with impatient customers, collisions and unreliable server. J. Siberian Federal Univ. Math. Phys. 13(2), 218-230 (2020)

6. Dudin, A.: Operations research perspectives. Oper. Res. 5, 245-255 (2018)

7. Haight, F.A.: Queueing with reneging. Metrika 2(1), 186-197 (1959)

8. Jain, M., Rani, S.: Markovian model of unreliable server retrial queue with discouragement. Proc. Natl. Acad. Sci. India Section A: Phys. Sci. 1-8 (2020)

9. Kim, C., Dudin, A., Dudina, O., Klimenok, V.: Analysis of queueing system with non-preemptive time limited service and impatient customers. Methodol. Comput. Appl. Probab. 1-32 (2019)

10. Kim, J.S.: Retrial queueing system with collision and impatience. Commun. Korean Math. Soc. 25(4), 647-653 (2010)

11. Kobayashi, H., Mark, B.L.: System Modeling and Analysis: Foundations of System Performance Evaluation. Pearson Education India (2009)

12. Kuki, A., Bérczes, T., Tóth, Á., Sztrik, J.: Numerical analysis of finite source Markov retrial system with non-reliable server, collision, and impatient customers. In: Annales Mathematicae et Informaticae, vol. 51, pp. 53-63. Liceum University Press (2020) 
13. Kumar, R., Som, B.K.: A multi-server queue with reverse balking and impatient customers. Pak. J. Stat. 36(2), 91-101 (2020)

14. Lakaour, L., Aissani, D., Aissanou, K., Barkaoui, K.: $M / M / 1$ retrial queue with collisions and transmission errors. Methodol. Comput. Appl. Probab. 1-12 (2018)

15. Law, A.M.: Statistical analysis of simulation output data: the practical state of the art. In: Proceedings of the 2015 Winter Simulation Conference, pp. 1810-1819. IEEE Press (2015)

16. Law, A.M., Kelton, W.D.: Simulation Modeling and Analysis. McGraw-Hill, New York (1991)

17. Lowndes, V., Berry, S.: Introduction to the use of queueing theory and simulation. In: Berry, S., Lowndes, V., Trovati, M. (eds.) Guide to Computational Modelling for Decision Processes. SFMA, pp. 145-171. Springer, Cham (2017). https://doi. org/10.1007/978-3-319-55417-4_5

18. Nazarov, A., Sztrik, J., Kvach, A.: A survey of recent results in finite-source retrial queues with collisions. In: Dudin, A., Nazarov, A., Moiseev, A. (eds.) ITMM/WRQ -2018. CCIS, vol. 912, pp. 1-15. Springer, Cham (2018). https://doi.org/10.1007/ 978-3-319-97595-5_1

19. Rubinstein, R.Y., Kroese, D.P.: Simulation and the Monte Carlo Method. Wiley, Hoboken (2016)

20. Satin, Y., Zeifman, A., Sipin, A., Ammar, S., Sztrik, J.: On probability characteristics for a class of queueing models with impatient customers. Mathematics 8(4), 594 (2020)

21. Tóth, Á., Bérczes, T., Sztrik, J., Kvach, A.: Simulation of finite-source retrial queueing systems with collisions and non-reliable server. In: Vishnevskiy, V.M., Samouylov, K.E., Kozyrev, D.V. (eds.) DCCN 2017. CCIS, vol. 700, pp. 146-158. Springer, Cham (2017). https://doi.org/10.1007/978-3-319-66836-9_13

22. Tóth, Á., Sztrik, J.: Simulation of finite-source retrial queuing systems with collisions, non-reliable server and impatient customers in the orbit. In: Proceedings of 11th International Conference on Applied Informatics, Eger, Hungary. CEUR Workshop Proceedings (CEUR-WS.org), vol. 2650, pp. 408-419 (2020). http:// ceur-ws.org/Vol-2650/

23. Wehrle, K., Günes, M., Gross, J.: Modeling and Tools for Network Simulation. Springer, Heidelberg (2010). https://doi.org/10.1007/978-3-642-12331-3

24. $\mathrm{Wu}, \mathrm{H}$., He, Q.M.: Double-sided queues with marked Markovian arrival processes and abandonment. Stoch. Models 1-36 (2020) 